\section{A pilot trial of deferiprone in pantothenate kinase-associated neurodegeneration patients}

\author{
Mohammad Rohani, Saeed Razmeh, \\ Gholam Ali Shahidi, Elham Alizadeh, \\ Maryam Orooji \\ Iran University of Medical Sciences, \\ Tehran, Iran
}

\begin{abstract}
Pantothenate kinase-associated neurodegeneration (PKAN) is the most common form of neurodegeneration with brain iron accumulation, it is an autosomal recessive disease due to mutation in PANK 2 on chromosome 20, which causes the accumulation of iron in basal ganglia and production of free radicals that cause degeneration of the cells. Deferiprone is an iron chelator that was used in treatment of thalassemia patients, it can cross the blood-brain barrier and reverse the iron deposition in the brain. Five patients with genetically confirmed PKAN received $15 \mathrm{mg} / \mathrm{kg}$ deferiprone twice daily. All patients were examined at baseline, 12 and 18 months and magnetic resonance imaging (MRI) was done at the baseline and after 18 months. In our study qualitative evaluation of MRI showed that deferiprone was able to reduce the iron load in globus pallidus of all the patients and the results of clinical rating scales show that in four patients, there is an improvement in the
\end{abstract}

first 12 months. The results of our paper show that deferiprone can prevent the progression of the disease.

\section{Introduction}

Neurodegeneration with brain iron accumulation (NBIA), which was called previously Hallervorden-Spatz syndrome, includes the growing number of neurodegenerative disorders characterized by iron accumulation in the brain, especially basal ganglia. ${ }^{1}$ The clinical manifestations of NBIA are various, including extrapyramidal movement disorders (dystonia, Parkinsonism, choreoathetosis), intellectual dysfunction, spasticity, visual disturbance and bulbar dysfunction. NBIAs include pantothenate kinase-associated neurodegeneration (PKAN), neuroferritinopathy (NFT), aceruloplasminaemia, Kufor-Rakeb disease (KRD), mitochondrial-membrane proteinassociated neurodegeneration (MPAN), betapropeller protein-associated neurodegeneration (BPAN), PLA2G6-associated neurodegeneration (PLAN), fatty acid hydroxylaseassociated neurodegeneration (FAHN), COASY protein-associated neurodegeneration (CoPan), and Woodhouse-Sakati syndrome. ${ }^{2-4}$ The major form of NBIA is PKAN, which is associated with mutations in the pantothenate kinase gene. It is separated into classic and atypical forms. Individuals with a classic disease have a more rapid progression of symptoms with earlier onset. In most cases, the atypical disease progresses slowly
Correspondence: Saeed Razmeh, Iran University of Medical sciences, Tehran, Iran. E-mail: srazmeh@yahoo.com

Key words: Deferiprone; Pantothenate kinaseassociated neurodegeneration; Neurodegeneration with brain iron accumulation.

Received for publication: 25 June 2017.

Revision received: 16 November 2017.

Accepted for publication: 16 November 2017.

This work is licensed under a Creative Commons Attribution NonCommercial 4.0 License (CC BY-NC 4.0).

(C) Copyright M. Rohani et al., 2017

Licensee PAGEPress, Italy

Neurology International 2017; 9:7279

doi:10.4081/ni.2017.7279

over several years, and sometimes decades.57 There is currently no suitable therapy for NBIA patients and different drugs including baclofen, clonazepam, trihexyphenidyl, levodopa and Botulinum toxin (BTX) often reduce the disability and increase the quality of life without effect on disease progression. Deferiprone is an iron chelator that was used in the treatment of iron overload of thalassemia patients, can cross the blood-brain barrier and decrease the iron that deposits in the brain.8,9 This study evaluates the efficacy of deferiprone based on clinical symptoms and brain magnetic resonance imaging (MRI) of PKAN patients.

Table 1. Patients' clinical presentations and imaging results.

\begin{tabular}{lccccc} 
Patient's number & Sex & Age (years) Duration of disease (years) & \multicolumn{2}{c}{ Clinical presentations } \\
1 & F & 29 & 15 & Generalized dystonia, Parkinsonism & Tiger's eye sign \\
2 & F & 35 & 20 & Generalized dystonia, & Parkinsonism, dysarthria \\
\hline 3 & M & 24 & 17 & $\begin{array}{c}\text { Generalized dystonia, } \\
\text { Parkinsonism, dysarthria }\end{array}$ & Tiger's eye sign \\
4 & M & 32 & 7 & Generalized dystonia, Parkinsonism, & Tiger's eye sign \\
\hline 5 & M & 23 & 10 & Gensarthria & Tiger's eye sign \\
\hline
\end{tabular}

Table 2. Unified Parkinson's Disease Rating Scale and Burke-Fahn-Marsden's dystonia rating scores together with blinded video rating.

\begin{tabular}{|c|c|c|c|c|c|c|c|c|}
\hline \multirow[t]{2}{*}{ Patient's number } & \multicolumn{3}{|c|}{ UPDRS Motor score } & \multicolumn{3}{|c|}{ BFM score } & \multicolumn{2}{|c|}{ Blinded video rating } \\
\hline & Baseline & 12 months & 18months & Baseline & 12 months & 18months & 12 months & 18 months \\
\hline 1 & 15 & 12 & 12 & 39 & 32 & 32 & Moderately improved & No improvement \\
\hline 2 & 16 & 15 & 18 & 39 & 41 & 43 & Slightly improved & No improvement \\
\hline 3 & 37 & 37 & 37 & 75 & 72 & 72 & Slightly improved & No improvement \\
\hline 4 & 18 & 16 & 17 & 34 & 32 & 32 & Slightly improved & No improvement \\
\hline 5 & 33 & 35 & 37 & 68 & 70 & 70 & Unchanged & Worsen \\
\hline
\end{tabular}

UPDRS, Unified Parkinson's Disease Rating Scale; BFM, Burke-Fahn-Marsden. 


\section{Materials and Methods}

Seven patients whose diagnosis were confirmed according to a neurological examination, brain MRI and genetic test, with informed consent, were selected. The inclusion criteria consisted of patients whose disease has been confirmed by genetic testing over ten years. The exclusion criteria consisted of inability to perform MRI, sensitivity to deferiprone, cytopenia, lack of consent to participate in the study, heart problems, kidney disease $(\mathrm{cr}>1.5)$ and hepatic disease (ALT $>300$ ). This study was approved by the research ethics committee of Iran University of Medical Sciences. At baseline, all patients were examined on the basis of Unified Parkinson's Disease Rating Scale (UPDRS/III-Motor Section) and the BurkeFahn-Marsden (BFM) scale for dystonia. Patients were also blindly evaluated through videotaping and brain MRI susceptibility weighted imaging (SWI) was used to analyse brain iron. Then, the deferiprone tablet was prescribed at $30 \mathrm{mg} / \mathrm{kg}$ daily, and all the patients were checked for the possible side effects of drug, complete blood count (CBC) weekly, and liver function tests (LFT), blood urea nitrogen (BUN), creatinine, serum iron, ferritin, calcium, and phosphorus monthly. All patients were evaluated according to mentioned scores at 6,12 and 18 months in the movements disorder clinic by an independent expert neurologist. All patients underwent brain MRI at baseline and 18 months later at the end of the study in one center, and the protocol of SWI for morphologic assessment was done and analyzed blindly by two expert radiologists qualitatively. The SPSS 22 was used for statistical analyses and $\mathrm{P}<0.05$ was considered as a significant level. The differences between the means of these parameters were tested by using repeated ANOVA measures.

\section{Results and Discussion}

Seven patients were enrolled. However, two patients were excluded, one due to severe truncal dystonia and inability to do MRI and another one due to severe anemia and leukopenia 1 year after treatment. The other 5 patients ( 3 males and 2 females; age range 23-35 years, mean $\pm \mathrm{SD}=28.6 \pm 5.12$ years) were evaluated during the 18 -month treatment.

All the patients had eye of the tiger sign on brain MRI, and were positive for the PANK2 mutation. The clinical evaluation of the patients that was done according to
Unified Parkinson's Disease Rating Scale (UPDRS) and BFM score represented the improvements in the motor signs and symptoms in 12 months in first 4 patients. This improvement was significant in patient 1 and mild in patients $2,3,4$. In patient 5 , no evident change was observed in the first 12 months and observed worsening of the scores in 18 months. Iron has an important role in cellular metabolism. The abnormal accumulation in the basal ganglia can induce oxidative stress and production of free radicals that cause progressive neurodegeneration and necrosis in this area. ${ }^{10,11}$ The use of deferiprone can decrease successfully this brain overload with clinical and radiological improvement. Cossu et al. in the pilot trial that assessed the safety of deferiprone in NBIA patients, showed that this treatment can be effective in reducing the iron load in brain imaging and improve the signs and symptoms associated with iron accumulation; ${ }^{12}$ also in another study in Friedreich ataxia patients, the deferiprone reduced brain iron accumulation. ${ }^{13}$ However, due to variability in the clinical presentation of PKAN and lack of relation between the clinical and radiological improvement, there are many questions about the effect of deferiprone on NBIA. In a study by Zorzi et al. evaluating the safety and efficacy of deferiprone in 9 PKAN patients, deferiprone was shown to be safe and without adverse side effects after one year of treatment. In this study, qualitative and quantitative evaluation of MRI showed that deferiprone was able to reduce the iron load in globus pallidus but did not show clinical improvement in all of the patients that were assessed by the BFM Dystonia Rating scales. ${ }^{14}$ Our study was done in 18 months, and, to our knowledge, this is the longest follow up pilot trial in PKAN patients (Tables 1 and 2).

\section{Conclusions}

Same as previous studies, in our study qualitative evaluation of SWI MRI showed reduction of iron load in globus pallidus in all patients. However, clinical improvement was seen in some patients in the first 12 months of study, without any change in the next 6 months, with worsening of signs and symptoms in 1 patient. The radiological improvement in contrast to any significant clinical improvement shows clinical manifestation depends on many factors including age, duration of disease, dose of deferiprone and the gap between the onset of the disease and start of treatment. In conclusion, according to of our pilot trial, deferiprone is safe in PKAN patients and can reduce the iron overload radiologically. Regarding the fact that most patients did not progress clinically and some patients improved, we think that deferiprone can be associated with neurological improvement, but it is essential that larger trials confirm our results with more patients, control group, other NBIA patients, and using different doses of deferiprone.

\section{References}

1. Thomas M, Jankovic J. Neurodegenerative disease and iron storage in the brain. Curr Opin Neurol 2004; 17:437-42.

2. Gregory A, Polster BJ, Hayflick SJ. Clinical and genetic delineation of neurodegeneration with brain iron accumulation. J Med Genet 2009;46:73-80.

3. Perry TL, Norman MG, Yong VW, et al. Hallervorden-Spatz disease: cysteine accumulation and cysteine dioxygenase deficiency in the globus pallidus. Ann Neurol 1985;18:4829.

4. Kurian MA, McNeill A, Lin JP, Maher ER. Childhood disorders of neurodegeneration with brain iron accumulation (NBIA). Dev Med Child Neurol 2011;53:394-404.

5. Hayflick SJ. Unraveling the Hallervorden-Spatz syndrome: pantothenate kinase-associated neurodegeneration is the name. Curr Opin Pediatr 2003;15:572-7.

6. Zhou B, Westaway SK, Levinson B, et al. A novel pantothenate kinase gene (PANK2) is defective in HallervordenSpatz syndrome. Nat Genet 2001;28:345-9.

7. Szanto J, Gallyas F. A study of iron metabolism in neuropsychiatric patients. Hallervorden-Spatz disease. Arch Neurol 1966;14:438-42.

8. Consky ES, Lang AE. Clinical assessments of patients with cervical dystonia. In: Jankovic J, Hallett M (eds.) Therapy with botulinum toxin. New York, NY: Marcel Dekker, Inc.; 1994. pp. 211-37.

9. Olivieri NF, Brittenham GM, McLaren $\mathrm{CE}$, et al. Long-term safety and effectiveness of iron-chelation therapy with deferiprone for thalassemia major. New Engl J Med 1998;339:417-23.

10. Connor JR, Menzies SL, Burdo JR, Boyer PJ. Iron and iron management proteins in neurobiology. Pediatr Neurol 2001;25;148-55.

11. Kakhlon O, Breuer B, Munnich A, Cabantchik ZI. neurological disorders associated with iron misdistribution: the therapeutic potential of siderophores. In: Gadoth N, Göbel H (eds.) Oxidative 
stress and free radical damage in neurology. Oxidative stress in applied basic research and clinical practice. New York, NY: Humana Press; 2011. pp. 169-90.

12. Cossu G, Abruzzese G, Matta G, et al. Efficacy and safety of deferiprone for the treatment of pantothenate kinase- associated neurodegeneration (PKAN) and neurodegeneration with brain iron accumulation (NBIA): results from a four years follow-up. Parkinsonism Relat Disord 2014;20:651-4.

13. Boddaert N, Le Quan Sang KH, Rotig A, et al. Selective iron chelation in Friedreich ataxia: biologic and clinical implications. Blood 2007;110:401-8.

14. Zorzi G, Zibordi F, Chiapparini L, et al. Iron-related MRI images in patients with Pantothenate Kinase-Associated Neurodegeneration (PKAN) treated with Deferiprone: results of a phase II pilot trial. Mov Disord 2011;26:1756-9. 